Journal of Thermophysics and Heat Transfer

Vol. 8, No. 3, July-Sept. 1994

\title{
Radiative Heat Transfer in Finite Cylindrical Enclosures with Nonhomogeneous Participating Media oy:
}

\author{
Pei-Feng Hsu* and Jerry C. Kut \\ Wayne State University, Detroil, Michigan 48202
}

\begin{abstract}
Results of a numerical solution for radiative heat transfer in homogeneous and nonhomogeneous participating media are presented. The geometry of interest is a finite axisymmetric cylindrical enclosure. The integral formulation for radiative transport is solved by the YIX method. A three-dimensional solution scheme is applied to two-dimensional axisymmetric geometry to simplify kernel calculations and to avoid difficultics associated with treating boundary conditions. As part of the etrort to improve modeling capabilities for turbulent jet diffusion flames, predicted distributions for flame temperature and soot volume fraction are used to calculate radiative heat transfer from soot particles in such flames. It is shown that the nonhomogeneity of radiative property has very significant effects. The peak value of the divergence of radiative heat flux could be underestimated by a factor of 7 if a mean homogeneous radiative property is used. Since recent studies have shown that scattering by soot agglomerates is significant in flames, the effect of magnitude of scattering is also investigated and found to be nonnegligible.
\end{abstract}

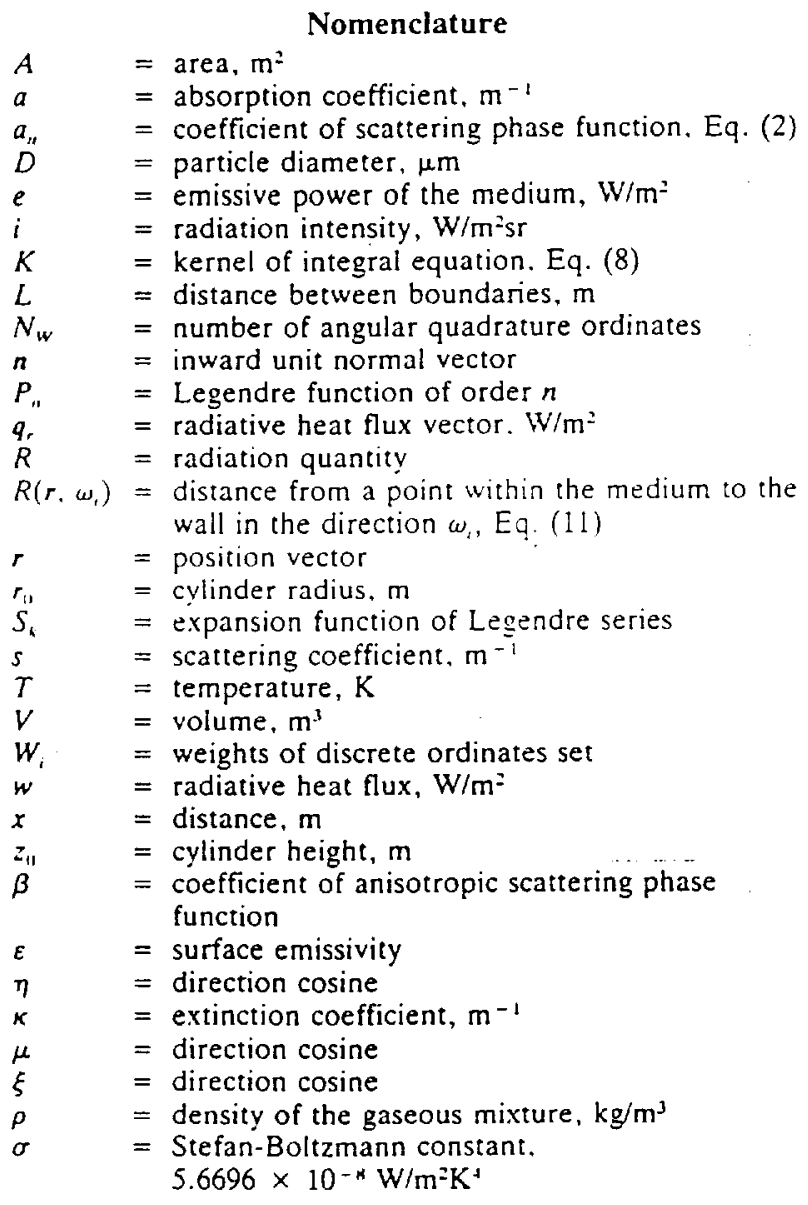

Received May 20. 1993; revision received Nov. 12. 1993; accepted for publication Nov. 22, 1993. Copyright 201994 by the American Institute of Aeronautics and Astronautics, Inc. All rights reserved.

- Research Associate. Mechanical Engineering Department; currently Assistant Professor. Mechanical and Aerospace Engineering Department. Florida Institute of Technology, Melbourne, FL $3290 \mathrm{t}$. Member AIAA.

†Associate Prolessor. Member AlAA.

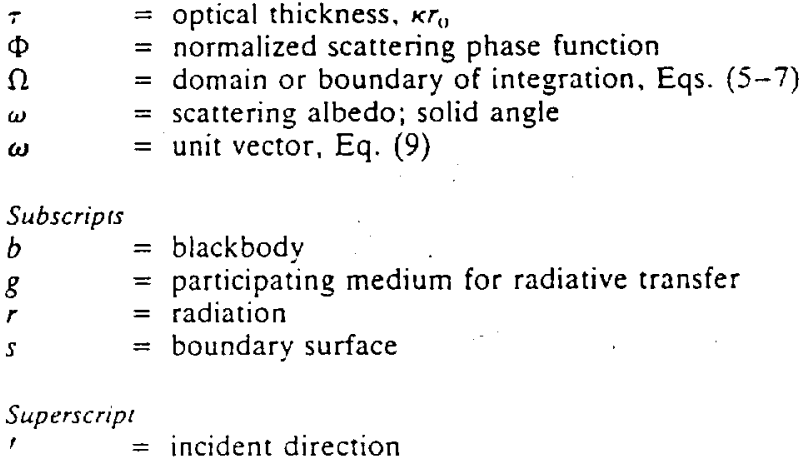

\section{Introduction}

D ADIATIVE heat transfer in a cylindrical enclosure with 12 a participating medium is a problem of practical importance, e.g., in the design of industrial fumaces and many combustion devices. A solution method that is accurate, efficient of both computing time and storage, flexible in a complex geometry, and compatible with the energy equation is needed for the prediction of radiative performance. Few current methods can satisfy all or part of these requirements. The Monte Carlo method is flexible and requires little storage, but can be extremely time-consuming, and the results are subject to statistical error. The zonal method and the finite element method are accurate but require large amounts of computer time and storage. It is also difficult to handle nonhomogeneity or anisotropic scattering using the zonal method. The product integration method,' while faster than the zonal and finite element methods, does not reduce the required storage. The discrete ordinates $\mathrm{Sn}$ method, although accurate and less demanding of memory for large grid systems. suffers from ray effects ${ }^{2}$ and high computer time for multidimensional combined mode heat transfer problems. To deal with multi. dimensional nonhomogeneous media, adaptive grid and adaptive difference schemes must be used with the discrete-ordinates method to maintain the same order of local error. This will consume large amounts of CPU time and memory, and make calculation using the $\left(S_{n}\right)$ method impractical. The spherical harmonics $(P n)$ method, "which needs high-order approximation to achieve accurate results for an optically thin medium, is tedious in formulation and also requires large amounts of computer time and memory in dealing with non- 
homogeneous media. Recently, the finite volume method has been applied to cylindrical geometry. ${ }^{+}$For problems involving fluid flow computation, the method can be formulated to use the same grid system. However, a rather involved discretization scheme must be used.

In this article, a recently developed numerical method, the YIX methods.t and its extension to three-dimensional geometry.' is used to solve the axisymmetric radiative transfer problem within turbulent jet diffusion flames. The formulation and solution scheme with cylindrical geometry (not limited to axisymmetric) is described in detail. Results are presented for homogeneous and nonhomogeneous participating media. The significance of the nonhomogeneity effect is demonstrated through comparisons with results for equivalent homogeneous media. The treatment of media with spectrally dependent radiative properties is not considered in this study, but will be a subject for a future paper.

There is increasing interest in the calculation of radiation heat transfer within nonhomogeneous sooting flames. ${ }^{-1 "}$ As part of the effort to couple turbulent diffusion flame modeling, soot formation and oxidation, and radiation heat transfer in a flame code, the treatment of radiation heat transfer within nonhomogeneous, absorbing, emitting, and scattering media is discussed.

\section{Mathematical Formulation}

The radiative heat transfer equation is written as

$$
\begin{aligned}
& \frac{\mathrm{d} i(r, \omega)}{\mathrm{d} l}=-\kappa i(r, \omega)+a i_{b}(r) \\
& +\frac{s}{4 \pi} \int_{\omega^{\prime}=+\pi} i\left(r, \omega^{\prime}\right) \Phi\left(\omega, \omega^{\prime}\right) \mathrm{d} \omega^{\prime}
\end{aligned}
$$

where the phase function, based on the Mie theory, can be expressed as':

$$
\Phi\left(\omega . \omega^{\prime}\right)=\sum_{n=11}^{N}(2 n+1) a_{n} P_{n}\left(\omega \cdot \omega^{\prime}\right)
$$

For particles with the size parameter $(\pi D / \lambda)$ much less than unity, the scattering effect is usually neglected, e.g., Rayleigh particles. $P_{,}\left(\omega \cdot \omega^{\prime}\right)$ can be expanded using the addition theorem of the Legendre function, ${ }^{13}$ and

$$
\omega \cdot \omega^{\prime}=\mu \mu^{\prime}+\xi \xi^{\prime}+\eta \eta^{\prime}
$$

where $(\mu, \xi, \eta)$ are the direction cosines of $\omega$ with respect to each coordinate axis. After the $P_{n}\left(\omega \cdot \omega^{\prime}\right)$ terms are expanded, the phase function can be expressed as

$$
\begin{aligned}
\Phi\left(\omega, \omega^{\prime}\right) & =\sum_{n=1}^{N}(2 n+1) a_{n} P_{n}\left(\omega \cdot \omega^{\prime}\right) \\
& =\sum_{k=1}^{\prime \prime} \beta_{k} S_{k}(\omega) S_{k}\left(\omega^{\prime}\right)
\end{aligned}
$$

where $\beta_{11}=1, \beta_{1}=\beta_{2}=\beta_{3}=3 a_{t}, \beta_{4}=5 a_{2} / 4, \beta_{5}=\beta_{0}=$ $5 a_{2} / 3, \beta_{7}=\beta_{x}=5 a_{2} / 12$. etc.

The integral formulation of radiative heat transfer in a general three-dimensional. gray, emitting, absorbing, and anisotropic scattering medium corresponding to Eq. (1) by Tan' is used here. Crosbie and Farrell't also developed similar integral expressions for intensity in three-dimensional cylindrical geometry. The present formulation is convenient to couple with the energy equation, since heat flux and its di- vergence are computed directly in addition to the computational efficiency for high-order scattering phase function

$$
\begin{aligned}
& 4 e_{g}(r)-\frac{1}{a} \nabla \cdot q_{r}(r) \\
& =\iiint_{\Omega} K\left(r, r^{\prime}\right)\left[\kappa e_{k}\left(r^{\prime}\right)-\frac{s}{4 a} \nabla \cdot q_{r}\left(r^{\prime}\right)\right] \mathrm{d} V\left(r^{\prime}\right) \\
& +\frac{s}{4} \sum_{k=1}^{M} \beta_{k} \iiint_{\Omega} K\left(r, r^{\prime}\right) w_{k}\left(r^{\prime}\right) S_{k}(\omega) d V\left(r^{\prime}\right) \\
& +\iint_{m} K\left(r, r^{\prime}\right)\left[e_{3}\left(r^{\prime}\right)-\frac{1-\varepsilon}{\varepsilon} q_{3}\left(r^{\prime}\right)\right] \\
& \times \cos \left(r-r^{\prime}, n^{\prime}\right) \mathrm{d} A\left(r^{\prime}\right) \quad r \in \Omega \\
& w_{i}(r)=\iiint_{\Omega} K\left(r, r^{\prime}\right)\left[\kappa e_{x}\left(r^{\prime}\right)-\frac{s}{4 a} \nabla \cdot q_{r}\left(r^{\prime}\right)\right] \\
& \times S_{i}(\omega) d V\left(r^{\prime}\right)+\frac{s}{4} \sum_{k=1}^{M} \beta_{k} \iiint_{n} K\left(r, r^{\prime}\right) w_{k}\left(r^{\prime}\right) \\
& \times S_{k}(\omega) S_{i}(\omega) \mathrm{d} V\left(r^{\prime}\right)+\iint_{i, \mathbf{l}} K\left(r, r^{\prime}\right) \\
& \times\left[e_{s}\left(r^{\prime}\right)-\frac{1-\varepsilon}{\varepsilon} q_{s}\left(r^{\prime}\right)\right] S_{i}(\omega) \cos \left(r-r^{\prime}, n^{\prime}\right) \mathrm{d} A\left(r^{\prime}\right) \\
& i=1,2, \ldots, M \quad r \in \Omega \\
& e_{s}(r)-\frac{1}{\varepsilon} q_{s}(r)=\iiint_{\Omega} K\left(r, r^{\prime}\right)\left[\kappa e_{R}\left(r^{\prime}\right)\right. \\
& \left.-\frac{s}{4 a} \nabla \cdot q_{r}\left(r^{\prime}\right)\right] \cos \left(r^{\prime}-r, n\right) d V\left(r^{\prime}\right) \\
& +\frac{s}{4} \sum_{k=1}^{M} \beta_{k} \iiint_{\Omega} K\left(r, r^{\prime}\right) w_{k}\left(r^{\prime}\right) S_{L}(\omega) \cos \left(r^{\prime}-r, n\right) \\
& \times \mathrm{d} V\left(r^{\prime}\right)+\iint_{\operatorname{dis} 2} K\left(r \cdot r^{\prime}\right)\left[e_{1}\left(r^{\prime}\right)-\frac{1-\varepsilon}{\varepsilon} q_{s}\left(r^{\prime}\right)\right] \\
& \times \cos \left(r-r^{\prime}, n^{\prime}\right) \cos \left(r^{\prime}-r \cdot n\right) \mathrm{d} A\left(r^{\prime}\right) \quad \cdot r \in \partial \Omega
\end{aligned}
$$

In the above equations, $e_{\mathrm{r}}$ and $e_{\mathrm{v}}$ are the blackbody emissive powers of the medium and the boundary, and $q$, is the net radiative heat flux at the wall. For isotropic scattering and nonscartering media, the second terms on the right sides of Eqs. $(5-7)$ can be deleted. The kernel $K$ is

$$
K\left(r, r^{\prime}\right)=\frac{\exp \left[-\int_{0}^{\left|r-r^{\prime}\right|} \kappa(r+\omega t) \mathrm{d} t\right]}{\pi\left|r-r^{\prime}\right|^{2}}
$$

and the unit vector

$$
\omega=\frac{r-r^{\prime}}{\left|r-r^{\prime}\right|}
$$

When the medium is nongray, the integral equations are essentially the same, except that all radiative quantities are wavelength-dependent, and $e_{g}$ and $e_{\text {, }}$ are replaced by the spectral Planck function.

\section{Numerical Method}

\section{Geometry}

Unlike planar two-dimensional $(x-y)$ geometry, which extends infinitely in the third $(z)$ direction, axisymmetric cylin. drical $(r-z)$ geometry does not extend infinitely in the third $(\theta)$ dimension. For the planar two-dimensional problem, by 


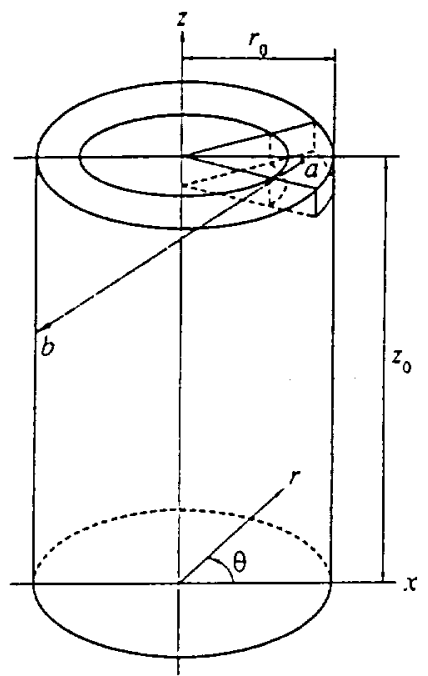

Fig. 1 Geometry of the cylindrical enclosure and coordinate system. Note the example ray of $a b$ originated from the volume node $a$ and hit boundary at point $b$. The direction of $\overline{a b}$ is determined from the discrete ordinates set used in angular quadrature.

integrating with respect to the infinite length direction in the volume integration in Eqs. (5-7), the Bickley functions of different degrees will be the kernels. However, if one performs the volume integrations with respect to $\theta$, as in the case of axisymmetry geometry (Fig. 1)

$$
\begin{aligned}
& \iiint_{12} K\left(r, r^{\prime}\right) w_{k}\left(r^{\prime}\right) S_{k}(\omega) \mathrm{d} V\left(r^{\prime}\right) \\
& =\int_{11}^{\prime \prime} \int_{0}^{2 \pi} \int_{11}^{r_{11}} K\left(r, r^{\prime}\right) w_{k}\left(r^{\prime}\right) S_{k}(\omega) r^{\prime} \mathrm{d} r^{\prime} \mathrm{d} \theta^{\prime} \mathrm{d} z^{\prime}
\end{aligned}
$$

depending on $S_{k}$, the integration related to $\theta$ can be expressed as

$$
\begin{aligned}
& \int_{1 \prime}^{=\pi} \frac{\exp \left[-\int_{11}^{i r-r^{\prime}} \kappa(r+\omega t) \mathrm{d} r\right]}{\pi\left|r-r^{\prime}\right|^{\prime \prime}} \mathrm{d} \theta^{\prime} \\
& \quad=\int_{11}^{2 \pi} \frac{\exp \left[-\kappa \sqrt{r^{2}+r^{\prime 2}-2 r r^{\prime} \cos \theta^{\prime}+\left(z-z^{\prime}\right)}\right]}{\pi\left[r^{2}+r^{\prime 2}-2 r r^{\prime} \cos \theta^{\prime}+\left(z-z^{\prime}\right)^{\prime \prime \prime}\right]^{\prime \prime}} \mathrm{d} \theta^{\prime}
\end{aligned}
$$

where $r$ and $r^{\prime}$ are any two points within the cylinder, and $\theta^{\prime}$ is the angle between $r$ and $r$ projected on the $x-y$ plane. Equation (10) is a kernel function different from the Bickley function. The kernel function is a function of three variables $\left(r . r^{\prime}\right.$. and $\left.z-z^{\prime}\right)$, which makes it difficult to tabulate or approximate by any computationally efficient function. Although direct numerical integration is possible, the amount of computation involved makes it essentially equivalent to a three-dimensional calculation. Another approach is to assume that the grid points and angular quadrature ordinates are predetermined, so that the new kernel function can be reduced to a single variable. The YIX integration points can then be precalculated and stored. However, the significantly larger number of YIX integration points will also make the computation equivalent to a three-dimensional calculation.

Other difficulties in treating axisymmetric cylinder cases as a iwo-dimensional problem are 1) an artificial symmetry boundary condition must be imposed at $r=0$ (Menguc and Viskanta. ${ }^{15}$ and Jamaluddin and Smith $\left.{ }^{10}\right)$, which could result in singularity caused by $1 / r$-term ${ }^{15}$; and 2 ) how to account for the curvature of the wall at $r=r_{i n}$, rather than treating it as a flat wall. ${ }^{15}$ These difficulties are eliminated in three-dimensional schemes.

\section{Numerical Quadratures}

The integrations of Eqs. (5-7) are performed using the YIX method. ${ }^{5}$ The integral equations are first rewritten into the distance-angular form. To maintain the same order of accuracy in angular integration at volume and boundary elements in three-dimensional geometries, the fully symmetric discrete ordinates and weights sets were used. "The use of discrete ordinates sets is discussed in Hsu et al. ${ }^{7}$

The volume and surface integrations on the right sides of Eqs. $(5-7)$ are constructed as follows:

$$
\begin{aligned}
& \iiint_{n} K\left(r, r^{\prime}\right) F\left(r^{\prime}\right) \mathrm{d} V\left(r^{\prime}\right) \\
& =\int_{d \pi} \frac{\mathrm{d} \omega}{\pi} \int_{0}^{\pi(r \cdot \omega)} \exp \left[-\int_{11}^{\prime} \kappa\left(r+\omega t^{\prime}\right) \mathrm{d} t^{\prime}\right] F(r+\omega t) \mathrm{d} t \\
& =\sum_{i}^{N_{w}^{*}} W_{1} \int_{10}^{R\left(r_{1} \omega_{i}\right)} \exp \left[-\int_{0}^{1} \kappa\left(r+\omega_{i} t^{\prime}\right) \mathrm{d} t^{\prime}\right] F\left(r+\omega_{i} t\right) d t
\end{aligned}
$$

$$
\begin{gathered}
\iint_{\partial \Omega} K\left(r, r^{\prime}\right) F\left(r^{\prime}\right) \cos \left(r-r^{\prime}, n\right) \mathrm{d} A\left(r^{\prime}\right) \\
=\int_{0}^{4 \pi} \exp \left[-\int_{0}^{l} \kappa\left(r+\omega t^{\prime}\right) \mathrm{d} t^{\prime}\right] F(r+\omega R) \frac{\mathrm{d} \omega}{\pi} \\
\approx \sum_{i}^{N_{w}^{*}} W_{i} \exp \left[-\int_{0}^{\prime} \kappa\left(r+\omega_{i} t^{\prime}\right) \mathrm{d} t^{\prime}\right] F\left(r+\omega_{i} R\right) \\
N_{w}^{*}=N_{w,} \quad \text { if } \quad r \in \Omega, \quad \text { or } \\
N_{w}^{*}=N_{w / 2,} \quad \text { if } \quad r \in \partial \Omega
\end{gathered}
$$

where the $R(r, \omega)$ is defined as

$$
\begin{aligned}
R(r, \omega)= & \min _{r+\omega+s, 1)}|r+\omega| \mid \\
= & \text { length of a beam emitted from } r \text { in } \omega \text { direction } \\
& \text { and striking the nearest boundary }
\end{aligned}
$$

$N_{w}$ is the number of ordinates, which depends on the order of discrete ordinate sets used. For the $S_{n}$ discrete ordinate set, $N_{w}=n(n+2)$. The distance integrals in Eq. (11) are evaluated using the YIX quadrature. It is interesting to note that the right sides of Eqs. (5) and (6) are essentially the same except for the additional $S_{i}$ term in Eq. (6). Therefore, the integrations in Eq. (6) can be avoided and a significant reduction of computational time can be achieved. For calculation with a high-degree anisotropic scattering phase function, this causes only a minor increase in CPU time [due to the second term on the right side of Eq. (5)] compared with other methods. This is impossible for the two-dimensional formulation, where different kernels exist in Eqs. (5) and (6). Additionally, the current scheme is flexibile enough to use multiple discrete ordinates sets in the formulation. which is very advantageous in dealing with ray effects. ${ }^{7}$ This flexibility does not exist in the conventional discrete ordinates method".is for the differential-integral formulation of the radiative transfer equation, where a consistent discrete ordinate set must be used even in the homogeneous case. By utilizing the axisymmetric geometry, only the first "wedge" of the boundary and volume elements is calculated (Fig. 1). To reduce computational time further, the angular quadrature is not carried out for all $N_{w}$ rays: in practice, only the rays with positive $y$ ordinates are calculated, due to the symmetry. The current scheme thus has the computational advantages of the three- 
dimensional scheme, but at a cost approaching that of the two-dimensional scheme.

\section{Solution Procedure}

The discretized integrals of Eqs. (5-7) are solved by iteration. The steps are 1) give an initial guess for $\nabla \cdot q_{r}, w_{k}(r)$. and $q_{s}(r)$, with $e_{\mathrm{q}}(r)$, and $e_{s}(r)$ known; 2$)$ calculate integrals on the right side of Eqs. (5-7) by the YIX quadrature; 3 ) calculate the new $\nabla \cdot q_{r}, w_{k}(r)$, and $q_{s}(r)$; and 4$)$ go to step 2 unless the convergence criterion is satisfied.

For the case with $e_{v}(r)$, and $e_{s}(r)$ as the unknowns, $\nabla \cdot q$, is obtained from the given heat source term of the energy equation and $q_{v}(r)$ from the given flux boundary conditions. The iterative procedure is the same as above.

\section{Results}

The computation was performed on a Sun $4 / 690$ workstation. In all calculations, the first YIX integration point is 0.01 , except in the optically thin case, with overall optical thickness less than 0.1 , where a first integration point of 0.001 is used. Typical run times can be several seconds to several hundred seconds, depending on the solution accuracy required and the optical thickness of the problem. Run time will be discussed later for the examples shown in Figs. 2 and 3.

In Fig. 2, the YIX method is applied to a benchmark problem: a uniform temperature $\left(T_{t}\right)$ nonscattering medium enclosed in a black cold cylinder with unity radius and height equivalent to one diameter. The net surface heat flux $q_{s}$ (or, in this particular case, heat flux $q$, at $r=1$ ), by three different solutions is shown in the figure. The YIX results are very close to the "exact" solution by Dua and Cheng." The maximum differences between YIX results and the exact solution are 3.2 .1 .9 , and $9.6 \%$ for $\tau=5.0,1.0$, and 0.1 , respectively. The P3 solution by Sun. ${ }^{21}$ which is similar to that of Menguc and Viskanta, ${ }^{15}$ is reasonably accurate for $\tau=1$, except at large $z$, where it overpredicts. At low optical thickness, P3 has a large deviation from the exact solution. The finite volume method ${ }^{+}$results are nearly the same as the exact solutions and are not shown in the figure. The calculation time for the YIX method increases as the optical thickness increases. The CPU times for $-=5.0 .1 .0$, and 0.1 are 335,195 , and $32 \mathrm{~min}$ respectively. with 0.001 as the first integration point and $\$ 16$ as the angular quadrature.

Experimental cylindrical furnace data were available for comparison with several numerical solutions. The $0.9-\mathrm{m}$ diam

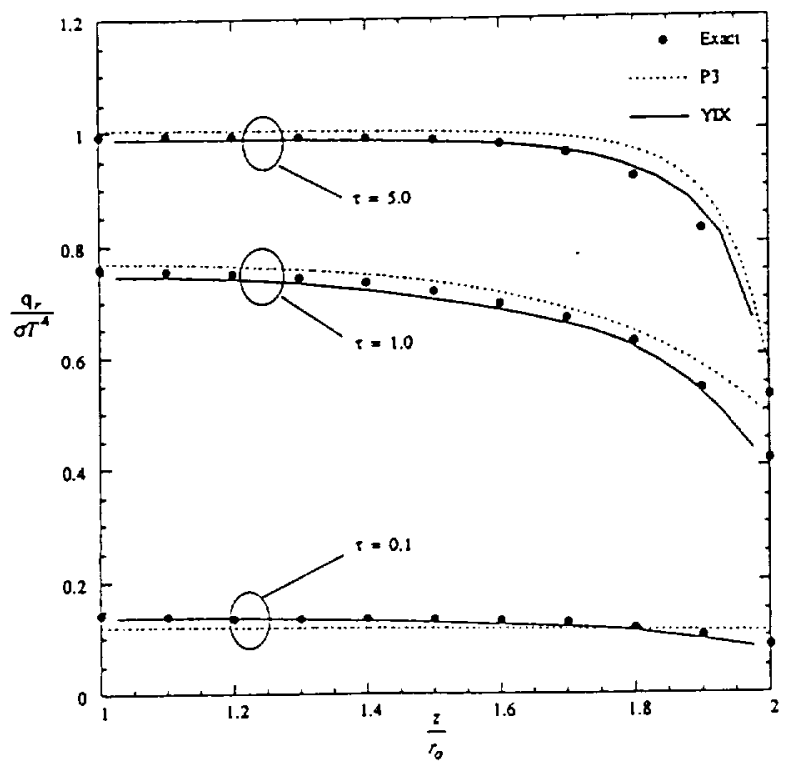

Fig. 2 Nondimensional radiant heat flux on the wall of cylindrical enclosure containing nonscattering medium at three different optical thicknesses.

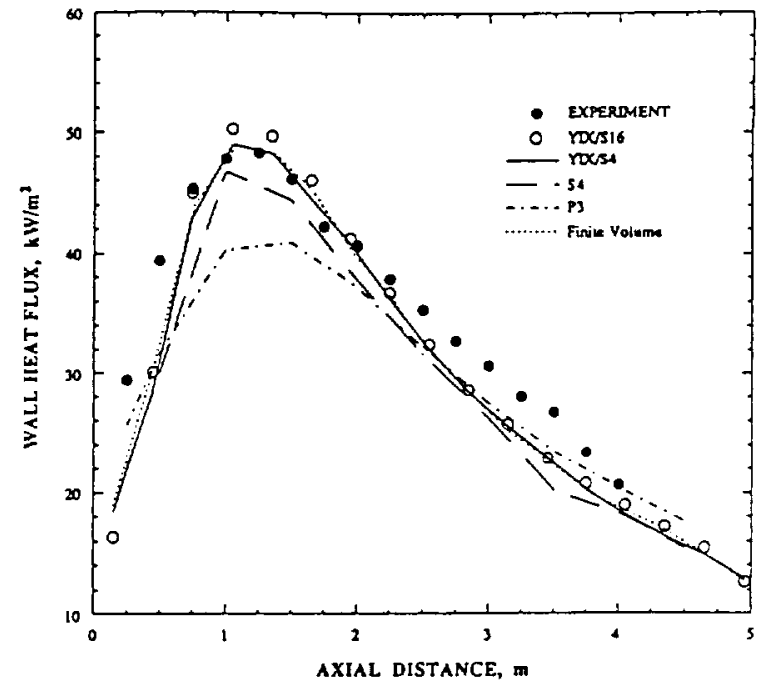

Fig. 3 Comparison of local surface heat fluxes at the side wall of a cylindrical furnace with experimental data.

and 5-m length water-cooled Delft furnace data were obtained from Jamaluddin and Smith ${ }^{16}$ and $W u$ and Fricker." The radiative medium for this experiment was modeled as gray, nonscattering with constant extinction coefficient of $0.3 \mathrm{~m}^{-1}$. The boundaries are at $425 \mathrm{~K}$ with an emissivity of 0.8 , except at the inlet and exit plenums, where the boundaries are treated as black surfaces at $300 \mathrm{~K}$. Figure 3 shows the measured wall heat flux and calculations by various methods. All the numerical solutions correctly predict the location of maximum heat flux. However, the P3 method"s seriously underpredicts the value of maximum heat flux, while other methods are reasonably close to each other. In the legend in Fig. 3. YIXJ $S_{n}$ represents the YIX quadrature using 0.001 as the first integration point and an angular quadrature using the $S n$ discrete ordinates set. It is interesting to note that the YIX/S4 and finite volumet methods produce nearly identical results. The higher-order quadrature (YIX/S16) predicts a higher peak flux than all other methods at an axial distance $1 \mathrm{~m}$ from the furnace base. Compared with results from the YIX and finite volume methods, the $S 4$ result $2=$ is lower for almost the entire length of the furnace. It is acknowledged $d^{+15: 22}$ that the measured values cannot be reproduced even with more accurate numerical methods. One of the reasons for the discrepancy is believed to be the assumption of a uniform extinction coefficient. This shows the necessity for treating the medium as nonhomogeneous in radiation transfer calculation for combustion systems.

For the experimental furnace problem, the YIX run times and maximum errors for using different integration points and. angular quadrature orders are shown in Table 1 . Note that the S16/0.001 case is used as the baseline for error comparison. As expected, with the higher-order discrete ordinates set, the CPU time increases in proportion to the corresponding $N_{w}$. While the smaller first integration point used in the YIX quadrature reduces the integration error, it also increases the CPU time.

As part of the effort to model a turbulent jet diffusion sooting flame, the radiation heat transfer within such a system is treated rigorously. Flame temperature (Fig. 4) and soor volume fraction data are obtained from numerical results for a turbulent ethylene jet diffusion flame in quiescent air with a nozzle diameter of $D=0.58 \mathrm{~mm}$ and a fuel flow rate of $3.96 \mathrm{~cm}^{3 / \mathrm{s}}$. $^{23}$ Since the extinction coefficient of soot aggregates can be approximated by that of Rayleigh spheres, ${ }^{24}$ it can be calculated from volume fraction with known soot refractive index and wavelength. We chose $m=1.7-i 0.7$ and $0.5-$ $\mu \mathrm{m}$ wavelength for this study. We are not claiming that the medium should be assumed gray. Rather, we do this so that 
Table I Comparison of CPU time and maximum error for the example problem

\begin{tabular}{|c|c|c|c|c|}
\hline \multirow[b]{2}{*}{$\begin{array}{l}\text { The first YIX } \\
\text { integration point }\end{array}$} & \multicolumn{2}{|c|}{$\mathrm{S} 16$} & \multicolumn{2}{|c|}{ St } \\
\hline & $\begin{array}{l}\text { CPU } \\
\text { time.s }\end{array}$ & $\begin{array}{l}\text { Maximum } \\
\text { error, \% }\end{array}$ & $\begin{array}{l}\text { CPU } \\
\text { time, s }\end{array}$ & $\begin{array}{c}\text { Maximum } \\
\text { error, \% }\end{array}$ \\
\hline $\begin{array}{l}0.001 \\
0.01\end{array}$ & $\begin{array}{l}270 \\
37.4\end{array}$ & $\begin{array}{l}0 \text { (buse case) } \\
4.7\end{array}$ & $\begin{array}{r}24.8 \\
3.8\end{array}$ & $\begin{array}{l}4.2 \\
5.9\end{array}$ \\
\hline
\end{tabular}

A $3 \times 17$ grid sysicm is used in all calculations on a Sun workstation.

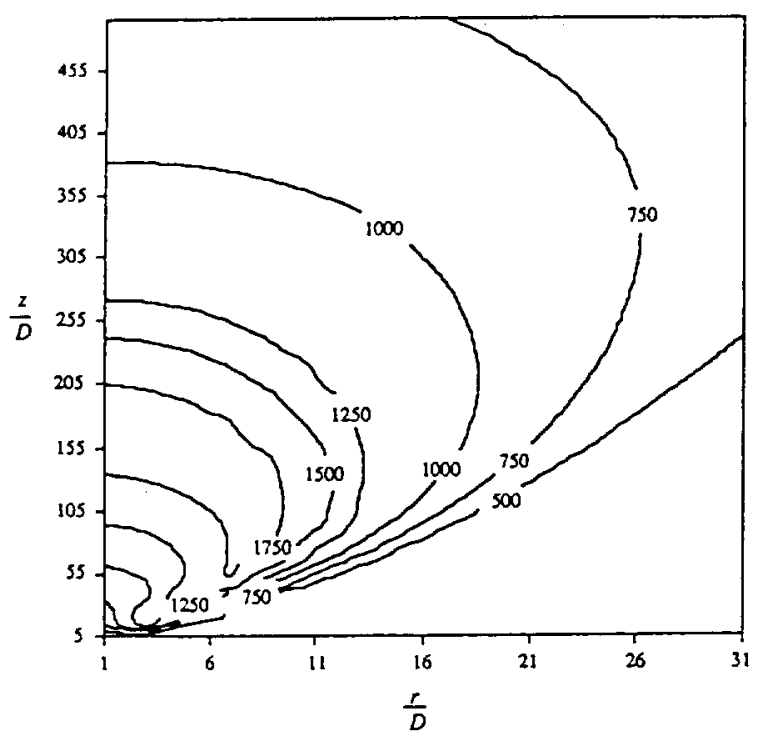

Fig. 4 Temperature (K) distribution of a turbulent jet diffusion flame.

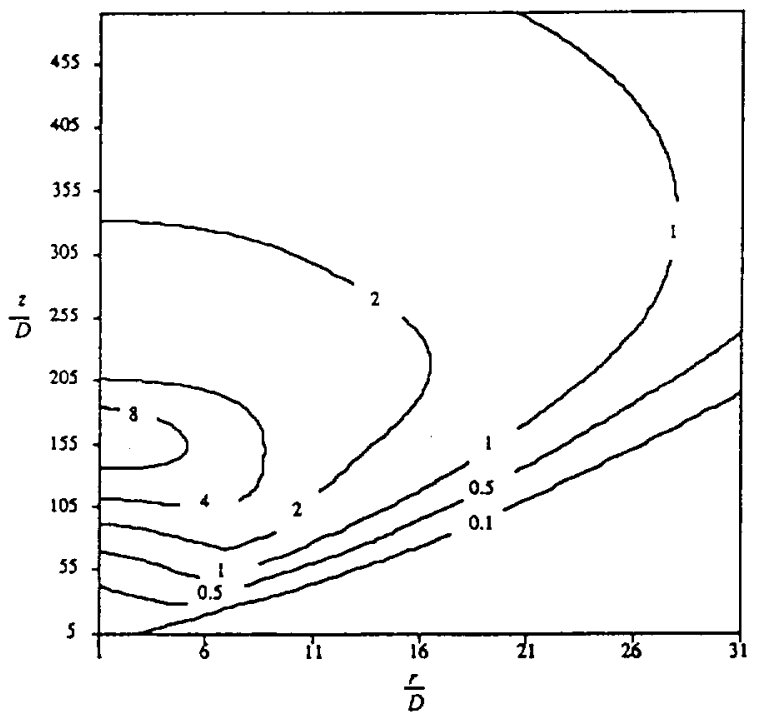

Fig. 5 Extinction coefficient $\left(m^{-1}\right)$ distribution of a turbulent jet diffusion name.

we can focus on effects of nonhomogeneity and scattering and be more computationally efficient. The local extinction coefficient, shown in Fig. 5, varies from as low as $10^{-13} \mathrm{~m}^{-1}$ to about $10 \mathrm{~m}^{-1}$ within the whole computational domain, which is much larger than the region covered in the figures. The computational domain is between $0 \leq r / D \leq 130$ and $0 \leq$ $z / D \leq 500$ using a $65 \times 50$ grid. The boundaries are assumed to be black at $300 \mathrm{~K}$. A high-order integration quadrature is used: the first YIX integration point of 0.001 and the $\$ 16$ discrete ordinates set.

We first calculate the nonscattering case. Based on the data of Figs. 4 and 5, the calculated normalized radiative heat flux

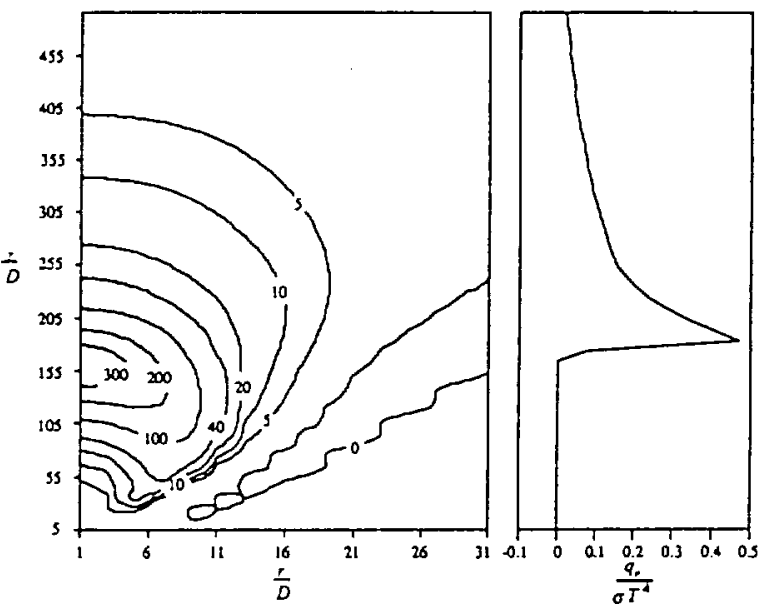

Fig. 6 Normalized radiative flux divergence contour and radial heat flux (at $r / D=31$ ) for turbulent diffusion flame with nonhomogeneous extinction coefficient and scattering albedo equals 0 .

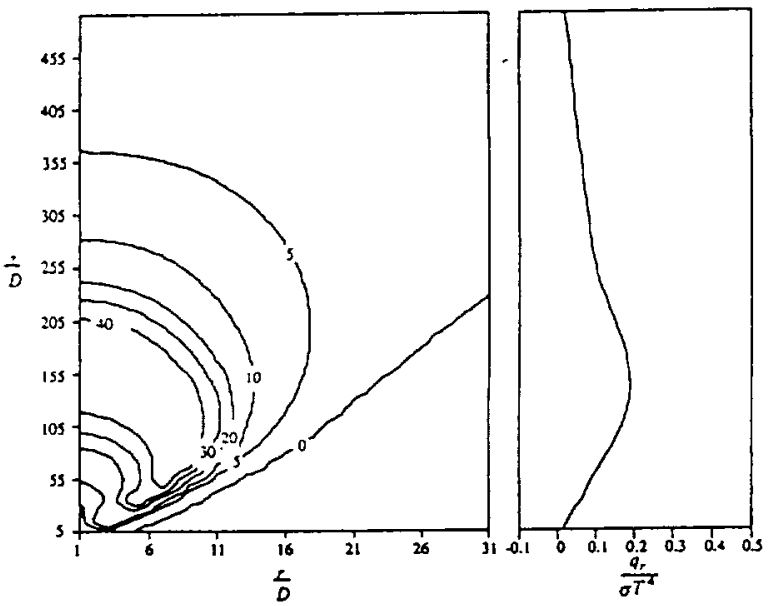

Fig. 7 Normalized radiative flux divergence contour and radial heat nux (at $r / D=31$ ) for turbulent diffusion name with homogeneous extinction coefficient and scattering albedo equals 0 .

divergence distribution is plotted along the $r-z$ axis (the left part of Fig. 6 ). The peak value $(\approx 370)$ of the normalized flux divergence occurs near the flame center. i.e., at $r / D=0$ and $z / D \approx 155$. The radiative heat flux is normalized with respect to the blackbody emissive power at $1000 \mathrm{~K}$. In Fig. 7 the same temperature data are used, but the extinction coefficient is treated as a constant. An equivalent mean extinction coefficient is obtained by averaging the local extinction coefficients of all the volume elements whose temperatures are higher than $300 \mathrm{~K}$. As shown in Fig. 7, the peak flux divergence is stiil at the flame center, but its value is reduced to about 49 from 370 , by a factor of more than 7 . In the right parts of Figs. $6-10$, the corresponding $q$, at $r / D=31$ for different conditions is also plotted. The homogeneous case (Fig. 7) has smooth $q$, distribution due to its constant extinction coefficient. On the other hand, nonhomogeneous cases have a sharp 


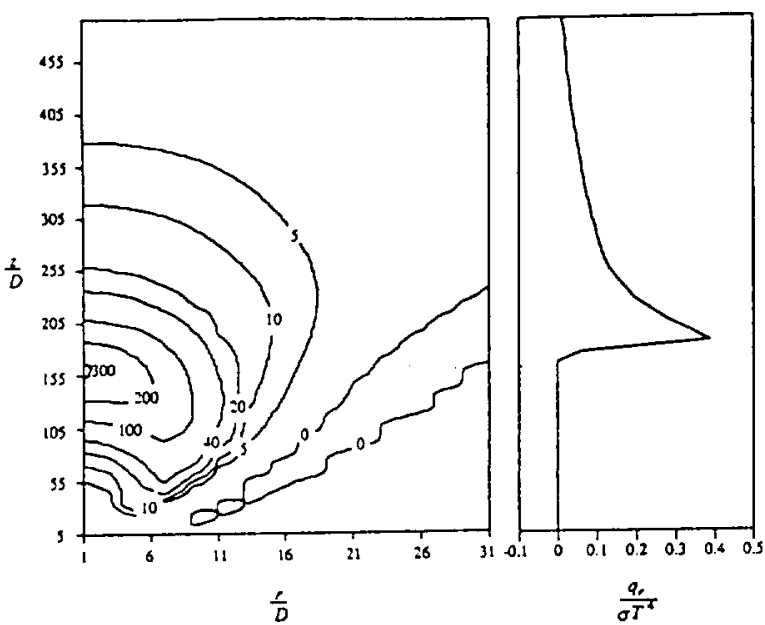

Fig. 8 Normalized radiative fux divergence contour and radial heat nux (at $r D=31$ ) for turbuient diffusion flame with nonhomogeneous extinction coefficient and scattering albedo equals 0.2 .

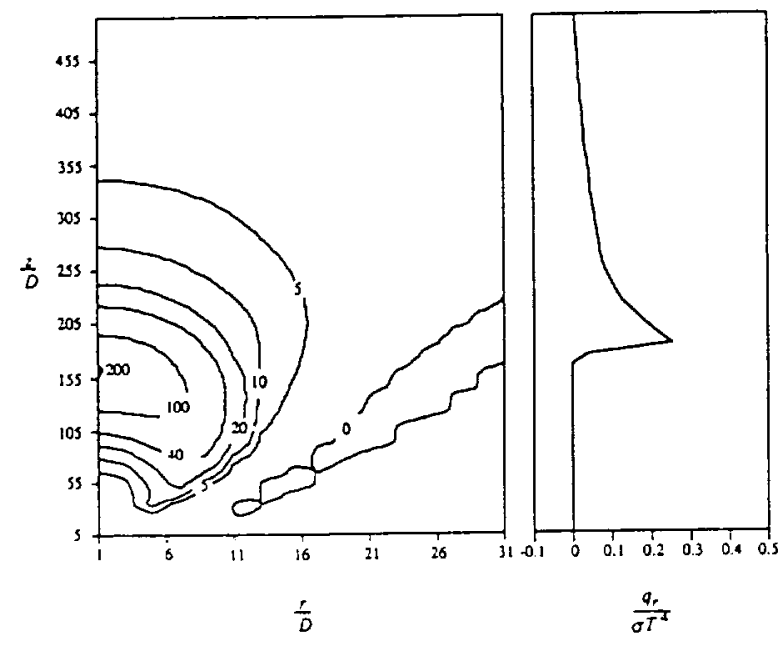

Fig. 9 Normalized radiative flux divergence contour and radial heat nux (at $r / D=31$ ) for turbulent diffusion name with nonhomogeneous extinction coefficient and scattering albedo equals 0.5 .

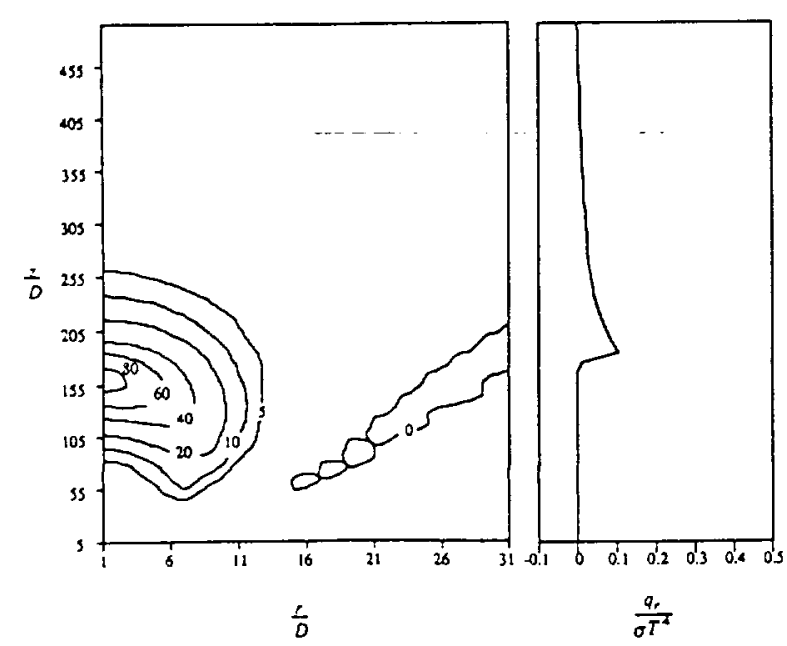

Fig. 10 Normalized radiative flux divergence contour and radial heat Mux (at $r / D=31$ ) for turbulent diffusion flame with nonhomogeneous extinction coefficient and scattering albedo equals 0.8 . peak $q_{r}$. It is evident that the effect of neglecting radiative property nonhomogeneity is very significant. To predict the flame behavior accurately with strong radiation effect and nonuniform soot distribution, it is necessary to model the radiation heat transfer by considering the local radiative property variation.

Figures 8-10 show the results under the same conditions as Figs. 4 and 5 , but with isotropic scattering added where the scattering albedo equals $0.2,0.5$, and 0.8 , respectively. Recent analysis $* 4$ and measurements"s both show that the scattering effect can be very significant, since soot particles are agglomerated in flames. Figure 8 shows a nearly identical flux divergence distribution as a nonscattering case (Fig. 6), except that the peak value is reduced to about 330 from 370 and the radial heat flux also decreases. As the scattering albedo increases, both radiative flux and its divergence decrease. Note that the scattering albedo may be a function of the position in the flame, since at later stages of the combustion (or at a higher position above the jet nozzle), the soot aggregates may grow larger, which in turn increases the scattering albedo. In the current numerical scheme using the YIX method, it is very easy to incorporate the distribution of the scattering albedo. However, since this data is not yet available, the calculation will be left for future study.

\section{Conclusions}

Numerical solutions of radiative heat transfer within a finite axisymmetric cylindrical enclosure involving homogeneous and nonhomogeneous media are treated rigorously. The use of the YIX quadrature and discrete ordinates is shown to be able to solve complicated multidimensional homogeneous as well as nonhomogeneous problems accurately. Numerical results for soot radiation from a turbulent jet diffusion flame show that neglecting the nonhomogeneity in radiative property can cause significant errors. They also show that the magnitude of scattering albedo has a significant effect on the radiative heat flux. It should be pointed out that the current numerical solution can also easily include nonuniform distribution of any other radiative properties, such as the scattering albedo.

\section{Acknowledgments}

Funding from NASA Lewis Research Center, Grant NAG31265 , is greatly appreciated. The authors also wish to thank Zhiqiang Tan for fruitful discussions of the application of the YIX method.

\section{References}

'Tan. Z., "Radiative Heat Transfer in Multidimensional Emitting. Absorbing, and Anisotropic Scattering Media-Mathematical Formulation and Numerical Methad." Journal of Heat Transfer, Vol. 111. No. 1, 1989, pp. 14t-147.

'Lathrop, K. D.. "Ray Effects in Discrete-Ordinates Equations." Nuclear Science and Engineering, Vol. 32. 1968. pp. 357-369.

${ }^{3}$ Menguc, M. P., and Viskanta. R.. "Radiative Transfer in Three. Dimensional Rectangular Enclosure." Journal of Quantitative Spec. troscopy and Radiative Transfer. Vol. 33, No. 6. 1985. pp. 533-549.

${ }^{+}$Chui, E. H. Raithby, G. D., and Hughes. P. M. j.. "Prediction of Radiative Transfer in Cylindrical Enclosure with the Finite Volume Method," Journal of Thermophysics and Heal Transfer, Vol. 6. No. 4. 1992. pp. 605-611.

sTan. Z., and Howell. J. R. . A New Numerical Method for Radiation Heat Transfer in Nonhomogeneous Participating Media." Journal of Thermophysics and Heat Transfer, Vol. 4. No. 4, 1990. Pp. $419-424$.

'Hsu, P.-F., Tan, Z., and Howell, J. R.. "A Correction of the YIX method for the Solution of Radiative Heat Transfer," unpublished, 1992.

'Hsu, P.-F., Tan, Z., and Howell. J. R., "Radiative Transfer by the YIX Method in Nonhomogeneous. Scattering. and Nongray Mle. dium." Journal of Thermophysics and Heat Transfer. Vol. 7. No. 3. 1993. pp. 487-495.

"Gore. J. P.. Ip. U.-S., and Sivathanu. Y. R.. "Coupled Structure and Radiation Analysis of Acelylene/Air Flames." Journal of Hear 
Transfer, Vol. 114, No. 2, 1992, pp. 487-493.

"Sivathanu, Y. R., Kounalakis, M. E., and Facth G. M "Soot and Continuum Radiation Statistics of Luminous Turbulent Diffusion Flames." Twenty-Third Symposium (International) on Combistion. Orleans, France, 1990, pp. 1543-1550.

"Fairweather, M., Jones, W. P., and Lindstedt, R. P., "Predictions of Radiative Transfer from a Turbulent Reacting Jet in a Cross Wind." Combustion and Flame, Vol. 89, No. 1. 1992, pp. 45-63.

"Siegel, R., and Howell, J. R., Thermal Radiation Heat Transfer. 3rd ed., Hemisphere. New York, 1993.

'EChu, C. M., and Churchill. S. W. ."Representation of an Angular Distribution of Radiation Scattered by a Spherical Particle," Journal of the Optical Society of America, Vol. 45. No. 11. 1955, pp. 958962.

"Gradshieyn. I. S.. and Ryzhick. I. M.. Table of Integrals. Series and Products. Academic Press. New York. 1980.

"Crosbie, A. L., and Farrell, J. B.. "Exact Formulation of Mlultiple Scattering in a Three-Dimensional Cylindrical Geometry," Jour nal of Quantitative Spectroscopy and Radianive Transfer. Vol. 31, No. 5. 1984. pp. $397-416$.

"Menguc, M. P.. and Viskanta, R., "Radiative Transfer in Axisymmetric. Finite Cylindrical Enclosure," Journal of Hear Transfer. Vol. 108, No. 2. 1986, pp. 271-276.

"Jamaluddin, A. S., and Smith, P. J. . "Predicting Radiative Transfer in Axisymmetric Cylindrical Enclosure Using the Discrete Ordinates Method, "Combustion Science and Technology, Vol. 62, 1988. pp. $173-186$.
"Tathrop, K. D., and Carlson, B. G., "Discrcte-Ordinates An. gular Quadrature of the Neutron Transpori Equation." Los Alamos Scientific Lab., Rept. LASL-3186. Los Alamos. NM. 1965.

"Fiveland, W. A., "Three-Dimensional Radiative Heat-Transfer Solutions by the Discrete-Ordinates Method." Journal of Thermophysics and Heal Transfer, Vol. 2. No. 4, 1988, pp. 309-316.

${ }^{4}$ Dua, S. S., and Cheng. P., "Multi-Dimensional Radiative Transfer in Non-Isothermal Cylindrical Media with Non-Isothermal Bounding Walls," International Journal of Heat and Mass Transfer. Vol. 18. 1975, pp. 245-259.

r'Sun. J., Ph.D. Dissertation. Mechanical Engineering Dept.. Wayne State Univ., Detroit, MI, in preparation.

"Wu. H. L.. and Fricker, N.. "The Characteristics of Swirl-Siabilized Natural Gas Flames-Part 2: The Behaviour of Swirling Jet

Flames in a Narrow Cylindrical Furnace." Journal of the Insirure of Fuel. Vol. 49. Sepl. 1976. pp. 144-15i.

:Fiveland. W. A.. " + Discrete Ordinates Method for Predicting Radiative Heat Transter in Axisymmetric Enclosures." American Society of Mechanical Engineers. Paper 82-HT-20. 1982

"Tong, L. Ph.D. Dissertation. Mechanical Engineering Dept. Wayne State Univ., Detroit. MI, in preparation.

${ }^{-}$Ku. J. C., and Shim. K.-H., "Optical Diagnostics and Radiative Properties of Simulated Soot Agglomerates." Journal of Heat Trans. fer, Vol. 113, No. 4, 1991, pp. 953-958.

"Koylu, U. O., and Faeth, G. M. " "Optical Properties of Overfire Soot in Buoyant Turbulent Diffusion Flames at Long Residence Times." Journal of Heas Transfer (to be published). 